\title{
The Role of Macrophages in the Pathogenesis of ALI/ARDS
}

\author{
Xiaofang Huang, ${ }^{1}$ Huiqing Xiu, ${ }^{1}$ Shufang Zhang, ${ }^{2}$ and Gensheng Zhang $\mathbb{D}^{1}$ \\ ${ }^{1}$ Department of Critical Care Medicine, Second Affiliated Hospital, Zhejiang University School of Medicine, Hangzhou, \\ Zhejiang 310009, China \\ ${ }^{2}$ Department of Cardiology, Second Affiliated Hospital, Zhejiang University School of Medicine, Hangzhou, Zhejiang 310009, China
}

Correspondence should be addressed to Gensheng Zhang; genshengzhang@zju.edu.cn

Received 12 January 2018; Revised 21 April 2018; Accepted 26 April 2018; Published 13 May 2018

Academic Editor: Teresa Zelante

Copyright (C) 2018 Xiaofang Huang et al. This is an open access article distributed under the Creative Commons Attribution License, which permits unrestricted use, distribution, and reproduction in any medium, provided the original work is properly cited.

\begin{abstract}
Despite development in the understanding of the pathogenesis of acute lung injury (ALI)/acute respiratory distress syndrome (ARDS), the underlying mechanism still needs to be elucidated. Apart from leukocytes and endothelial cells, macrophages are also essential for the process of the inflammatory response in ALI/ARDS. Notably, macrophages play a dual role of proinflammation and anti-inflammation based on the microenvironment in different pathological stages. In the acute phase of ALI/ARDS, resident alveolar macrophages, typically expressing the alternatively activated phenotype (M2), shift into the classically activated phenotype (M1) and release various potent proinflammatory mediators. In the later phase, the M1 phenotype of activated resident and recruited macrophages shifts back to the M2 phenotype for eliminating apoptotic cells and participating in fibrosis. In this review, we summarize the main subsets of macrophages and the associated signaling pathways in three different pathological phases of ALI/ARDS. According to the current literature, regulating the function of macrophages and monocytes might be a promising therapeutic strategy against ALI/ARDS.
\end{abstract}

\section{Introduction}

Acute lung injury (ALI)/acute respiratory distress syndrome (ARDS) is a devastating respiratory disorder, which leads to mortality in patients in intensive care units [1]. It is characterized by clinically significant hypoxemia, diffuse bilateral pulmonary infiltration, pulmonary edema, a decrease in pulmonary compliance, and a decrease in the functional residual capacity [2]. Pathological changes include increased vascular permeability caused by alveolar-capillary membrane dysfunction, with flooding of protein-rich fluid, alveolar hemorrhage, and fibrin deposition [3]. ALI/ARDS develops by excessive and uncontrolled systemic inflammatory responses to direct or indirect lung injury. Currently, there is increasing evidence suggesting that macrophages, including resident alveolar macrophages (AMs) and recruited macrophages from the blood, are key factors in the pathogenesis of ALI/ ARDS $[4,5]$. The role of macrophages during development of the inflammatory response is subtle. In general, they exert a proinflammatory effect in the early stage and exhibit an anti-inflammatory effect in the late stage. These effects may be attributed to the phenotypic transformation, which is in part regulated by the suppressor of cytokine signaling (SOCS) 1/SOCS3 and interferon regulatory factor (IRF) 4/ IRF5 [6-9]. In this review, we summarize the main subsets of macrophages involved in ALI/ARDS and the recent advances in the phenotypic and functional alterations. The identification of the cellular and molecular mechanisms associated with the role of macrophages in ALI/ARDS will provide a basis for some potential treatment strategies.

\section{Main Macrophages Involved in ALI/ARDS}

Classified by the responses to environmental stimuli, there are two polarization states of macrophages: the classically activated phenotype (M1) and the alternatively activated phenotype (M2) [10].

There are two main types of macrophages in the alveolus. The first type is the long-lived resident AMs, which are located at the air-tissue interface, with an approximate density of seven per alveolus [11]. As a predominant cell type in the alveolar airspaces, they act as a uniform, quiescent, and immunosuppressive population in the normal state $[12,13]$. The M2 phenotype is the main form of these 
resident AMs. The second type is the recruited AMs. When a stimulus occurs, such as in ALI/ARDS, peripheral blood monocytes are recruited into the alveolar lumen, where they differentiate into macrophages with the M1 phenotype $[11,14]$.

To further investigate the role of AMs in the pathogenesis of ALI/ARDS, clodronate-loaded liposomes have been used to eliminate AMs specifically in blockage experiments [15, 16]. Other studies have shown that the depletion of AMs significantly reduced pulmonary edema and ventilator-induced lung injury in rats $[17,18]$, which was partially attributed to the decreased recruitment of neutrophils in the lungs. Besides, depletion of AMs also has been found [19] to mitigate lung injury significantly at $4 \mathrm{~h}$ after lipopolysaccharide (LPS) administration in mice by attenuating neutrophilic alveolitis and reducing proinflammatory cytokines.

Machado-Aranda et al. [20] have shown that neutrophils were significantly recruited to the bronchoalveolar lavage fluid from $5 \mathrm{~h}$ after contusion-induced lung injury in the AM-depleted group, which resulted in a worsened pulmonary compliance, an increased lung permeability, and aggressively elevated proinflammatory cytokine levels. In a study by Broug-Holub et al. [21], substantial neutrophil recruitment and decreased bacterial clearance were found in the lungs of AM-depleted mice at $48 \mathrm{~h}$ after infection with Klebsiella pneumoniae. In addition, Narasaraju et al. [22] have shown that influenza infection led to excessive recruitment of neutrophils, extensive alveolar damage, and increased viral load in the AM-depleted group at 5 days after infection.

According to these results from various experimental models, AM depletion shows a protective effect against ALI/ARDS at an early stage, while it exacerbates lung injury at a later stage. Neutrophils are the first leukocytes recruited to the sites of inflammation in response to chemokines such as macrophage inflammatory protein-2 (MIP-2) and interleukin- (IL-) 8 released by activated AMs [23-26]. The accumulation of neutrophils is an important factor leading to tissue damage due to the overwhelming release of cytotoxic and proinflammatory mediators [27]. At an early stage, AM depletion may alleviate neutrophil-induced alveolitis by reducing inflammatory responses. At a later stage, circulating monocytes migrate into the interstitium or alveolar spaces in response to monocyte chemoattractant protein-1 (MCP-1) for pathogen clearance and phagocytosis of infected particles or other inflammatory debris (Figure 1(a)) [13, 28]. Macrophage phagocytosis of neutrophils and other apoptotic cells is an important step in the process of inflammatory response regulation. At this stage, the number of recruited AMs is increased, and the depletion of AMs may result in decreased phagocytic macrophages and control of inflammation. On the other hand, intraperitoneal administration of MCP-1 significantly increased the total number of macrophages as well as the number of phagocytizing neutrophils [29]. The increased chemokine ligand 2 (CCL-2 or MCP-1) expression in turn enhances CC chemokine receptor 2 (CCR2) expression in circulating monocytes and interstitial macrophages [30]. The interaction of CCL-2 with CCR2 plays an important role in the process of reepithelialization after lung injury
[31]. In mice subjected to intratracheal LPS treatment, CCR2 deficiency aggravated the apoptosis of alveolar epithelial cells and permeability injury [32]. Therefore, these two types of AMs act at an early or a late stage in the process of ALI/ ARDS: the resident AMs release cytokines for recruiting neutrophils or monocytes to promote and sustain inflammation, and the recruited AMs mainly function to clear pathogens and limit inflammatory responses.

Similarly, the role of circulating monocytes has been investigated in LPS-induced lung injury models. Herold et al. [32] have reported that depletion of circulating monocytes (the precursors of exudate macrophages) seems to aggravate LPS-induced lung injury. In contrast, Dhaliwal et al. [33] have found that depletion of the pool of peripheral blood monocytes might alleviate lung injury and might be a therapeutic target for ALI/ARDS. These controversial results may be due to the different times of monocyte depletion. In the study by Herold et al., depletion of circulating monocytes was performed at $12 \mathrm{~h}$ before LPS challenge [32]. Meanwhile, in the study by Dhaliwal et al., the process was initiated by the use of clodronate at $6 \mathrm{~h}$ after intratracheal LPS administration [33]. Taken together, AMs and peripheral blood monocytes exert distinctly exclusive functions at different stages of ALI/ARDS [34, 35]. The modulation of macrophages and monocytes may be a potential way to treat ALI/ARDS. However, the mechanism of cellular interactions between macrophages and several anti-inflammatory cells remains unclear [36].

\section{Phenotype and Functional Alternation of Macrophages in the Pathogenesis of ALI/ ARDS}

3.1. Exudative Phase of ALI/ARDS. In healthy lungs, resident AMs are highly plastic for immune responses, with relatively high expression levels of pathogen-associated molecular pattern and danger-associated molecular pattern receptors [37]. Upon stimulation in ALI/ARDS, resident AMs immediately shift to the predominant M1 phenotype in response to infection-induced activation of toll-like receptors (TLRs) or other recognition receptors [38]. These AMs act as the first line of defense against pathogenic microorganisms and lung injury, such as bacteria, endotoxins, viruses, and ventilatorinduced lung injury $[22,39,40]$, releasing various potent proinflammatory and deleterious mediators including IL$1 \beta$, IL-6, and IL-18 $[41,42]$. With these inflammatory factors, the neutrophils are recruited from the intravascular space, across the endothelium and epithelium, and finally into the lungs and alveolar space. It is widely known that excessive accumulation of proinflammatory cytokines and neutrophils is involved in the pathophysiology of tissue damage in inflammatory diseases [43]. Therefore, M1 macrophages serve as a promoter in the process of lung tissue damage in ALI/ARDS. However, a protective effect associated with M1 macrophages also has been detected. Recent studies have found that these macrophages generate a high level of amphiregulin $[44,45]$, a ligand of epithelial growth factor receptor, which has been found to protect the epithelial 


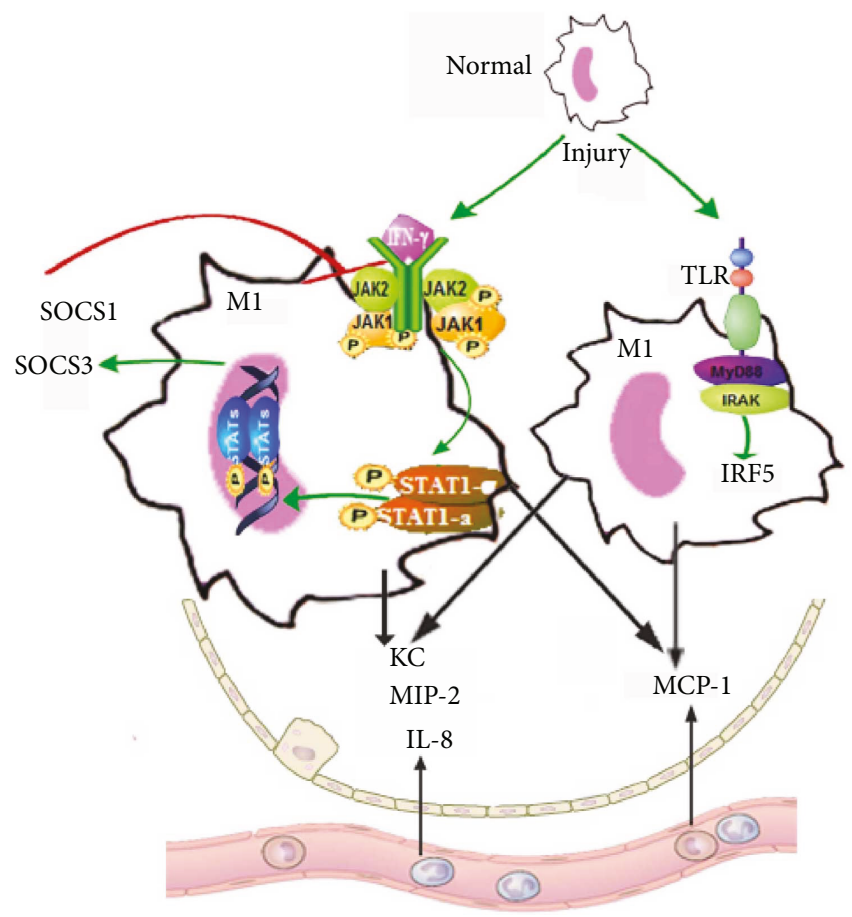

(a)



(b)

FIGURE 1: The molecular mechanism of SOCS- and IRF-regulated cytokine signaling in macrophages during ALI/ARDS. (a) Normal resident AMs are activated and shift into the M1 phenotype upon certain stimulation during the exudative phase of ALI/ARDS. Proinflammatory cytokines such as IFN- $\gamma$, TNF- $\alpha$, and IL- $\beta$ are excreted by M1 macrophages into the site of inflammation. The JAK-STAT1 pathway is activated by IFN $-\gamma$, and SOCS1 and SOCS3 are induced. SOCS1 and SOCS3 inhibit the signaling pathway by different mechanisms. IRF5 promotes M1 polarization by directly binding to IL-12 and IL-23 promoters. Leukocytes migrate into the cellular airspace by the activation of chemokines such as KC, MIP-2, and IL-8. Monocytes from the circulation are also recruited by chemokines such as MCP-1 and shift into the M1 phenotype. Crosstalk between SOCS3 and IRF5 may exist. (b) Macrophages shift from the M1 phenotype to the M2 phenotype during the later phase of ALI/ARDS. This process is regulated by several factors, including IL-4, IL-10, IL-13, STAT6, and IRF4. IL-4 or IL-13 activates the JAK-STAT6 pathway, and SOCS1 is induced. SOCS1 feedback inhibits the IL-4/IL-13 signaling. IRF4 inhibits IRF5 activation by a competing interaction with the adaptor MyD88. Recruited macrophages play an important role in eliminating apoptotic cells, debris, and pathogens. AMs: alveolar macrophages; IFN- $\gamma$ : interferon- $\gamma$; JAK: Janus kinase; IL: interleukin; STAT: signal transducer and activator of transcription; IRF: interferon regulator factor; SOCS: suppressors of cytokine signaling; KC: keratinocyte-derived chemokine; MIP: macrophage inflammatory protein; MCP: monocyte chemoattractant protein; TNF- $\alpha$ : tumor necrosis factor $\alpha$; TLR: toll-like receptor; MyD88: myeloid differentiation factor 88.

barrier and inhibit the gene expression of proinflammatory cytokines in LPS-induced ALI and ventilator-induced lung injury models $[44,45]$.

The shift of macrophage phenotypes is regulated by several signaling pathways (Figure 1(a)). M1 macrophages can be activated through the classical JAK/STAT1 pathway [46-48]. In detail, interferon- $\gamma$ (IFN- $\gamma$ ) binds to the receptors on the cell surface, activating Janus kinase 1 (JAK1), JAK2, and signal transducer and activator of transcription 1 (STAT1) [46]. In addition, STAT expression is negatively regulated, in part, via SOCS [49]. Under normal conditions, the expression levels of SOCS1 and SOCS3 are very low, but they are rapidly activated by IFN- $\gamma$ or LPS [6]. Specifically, SOCS1 and SOCS3 negatively regulate the JAK/ STAT pathway through binding with the key phosphorylated tyrosine residues of JAKs and/or cytokine receptors $[50,51]$. Besides, other possible mechanisms accounting for SOCS proteins inhibiting JAK/STAT signaling also have been identified $[52,53]$. In mouse macrophage cells, the downregulation of SOCS1 expression by SOCS1 short hairpin (sh) RNA transfection significantly increased the mRNA levels of JAK1 and STAT1 as well as promoted the polarization of macrophages to the M1 phenotype [54]. In addition, Qin et al. [55] have revealed that myeloidspecific SOCS3-deficient mice exhibit enhanced activities of STAT1/3 and increased plasma levels of proinflammatory cytokines and chemokines. Therefore, both SOCS1 and SOCS3 inhibit the polarization of macrophages into the M1 phenotype, decreasing proinflammatory chemoattractants. Additionally, M1 macrophages have shown an impaired proinflammatory effect and an enhanced anti-inflammatory effect in the absence of SOCS3 in a nephrotoxic nephritis model [7], thus indicating an essential role of SOCS3 in the development of M1 macrophages. However, whether similar mechanisms exist in ALI/ARDS models remains to be further investigated. 
The transcription factor IRF5 is another major regulator of proinflammatory M1 macrophage polarization. It is generally involved in the process of the downstream TLR-myeloid differentiation factor 88 signaling pathway, inducing proinflammatory cytokines and repressing transcription of antiinflammatory cytokines such as IL-10 [56]. In a study by Qin et al. [57], IRF5 expression was increased in the absence of SOCS3, which promoted M1 macrophage polarization. Thomas et al. [8] also have shown that IRF5 deficiency led to a lower proportion of M1 macrophage subsets in mice and a lower expression of M1-specific cytokines in human M1 macrophages [8]. Overall, STAT1, SOCS1/ SOCS3, and IRF5 are involved in the polarization of M1 macrophages, with substantial crosstalk among these signaling pathways [57-60].

3.2. Rehabilitation Phase of ALI/ARDS. After pathogenic factors are eliminated, resident and recruited macrophages shift from the M1 phenotype to the anti-inflammatory M2 phenotype [5]. According to various activating conditions, M2 macrophages are classified into four subtypes: M2a (IL-4- or IL-13-induced), M2b (immune complexes in combination with IL-1 $\beta$ or LPS), M2c (IL-10, transforming growth factor (TGF)- $\beta$, or glucocorticoids), and M2d (adenosine $A_{2 \mathrm{~A}}$ receptor agonists) [61-63]. M2 macrophages play an important role in lung tissue repair by limiting the levels of proinflammatory cytokines in the cellular space. They also help produce anti-inflammatory cytokines such as IL-10 and IL-1 receptor antagonist in response to T-helper 2 (Th2) cytokines [5, 35]. Apart from the balance of pro- and antiinflammatory cytokines, the clearance of neutrophils from inflammatory sites is another contributing factor for rehabilitation [64]. Once recruited, neutrophils exhibit apoptosis and the apoptotic cells accumulate [65]. With a potent phagocytic capacity, M2 macrophages remove the necrotic cells and debris. The elimination of apoptotic cells by phagocytes, known as efferocytosis, can activate anti-inflammatory signaling and terminate proinflammatory responses [66]. Phagocytosis of apoptotic neutrophils by M2 macrophages further increases the levels of IL-10 and TGF- $\beta 1[35,67]$, which may help to control inflammation. It also inhibits the expression of inducible nitric oxide synthase and stimulates the expression of arginase 1, thereby preventing reactive nitric oxide production [5]. Besides, M2-derived cytokines, including IL-4, IL-13, and IL-10, further enhance efferocytosis, with an increased level of mannose receptor expression $[68,69]$. With this positive feedback loop, efferocytosis and M2 polarization alleviate inflammation. However, it is still unclear how M2 differentiation is terminated and which mediators are involved.

In addition, recent studies have indicated that regulatory T cells (Tregs), a subset of $\mathrm{CD}^{+}$lymphocytes, are involved in the phenotypic transformation of macrophages $[70,71]$. Tregs may reduce lung inflammation by diminishing the elevation of macrophage proinflammatory cytokine levels and enhancing the efferocytosis of apoptotic neutrophils [72-74]. Taams et al. [74] have shown that human Tregs inhibit LPS-induced M1 monocyte proinflammatory responses and promote the M2 phenotype. Eliminating Tregs has resulted in sustained proinflammatory responses induced by LPS and reduced neutrophil apoptosis [73, 74]. Future studies investigating the possible mechanisms for Tregmediated neutrophil apoptosis are on the way [75].

Several signaling pathways are involved in the phenotypic shift from M1 to M2 macrophages (Figure 1(b)). The alternative macrophages are activated by Th2-type cytokines, including IL-4 and IL-13 [76]. However, the main sources of IL-4 and IL-13 in the body are largely unknown. Produced by various innate cells, IL- 4 and IL-13 have many similar effects on macrophages due to an identical receptor chain, IL- $4 \mathrm{R} \alpha$. Of note, IL-4 can activate the insulin receptor substrate- 2 signaling pathway through the $\gamma c$ chain, while IL-13 cannot [77]. Besides, IL-4 and/or IL-13 activate STAT6 and increase the expression of STAT6-responsive genes such as arginase 1 and 15-lipoxygenase [6]. In addition to the regulatory effects on the activation of macrophages by the classical IFN- $\gamma$ inducible pathway, SOCS1 regulates the alternative IL-4inducible pathway [78]. IL-4 induces SOCS1 expression in macrophages via STAT6 signaling, and SOCS1 feedback inhibits IL-4 signaling to limit the expression of STAT6responsive genes and the alternative macrophage activation pathway $[6,78]$. In a study by Liu et al. [7], SOCS3 suppression enhanced STAT3 activity; decreased the expression of IL-6, nitric oxide, and CD86; and increased the expression of mannose receptor and arginase, which promote the polarization of macrophages into the M2 phenotype. Their results suggest that the expression of SOCS3 maintains the activated phenotype M1 cells with proinflammatory properties and inhibits the phenotype with anti-inflammatory effects [7]. Taken together, SOCS1 and SOCS3 are involved in the classical macrophage activation pathways as well as in various alternative macrophage activation pathways. Further investigations to mitigate ALI/ARDS by regulating the balance between anti-inflammatory and proinflammatory effects of macrophages are needed.

IRF4, another member of the IRF family, has diverse effects in different situations. It inhibits IRF5 activation by a competing interaction with the adaptor myeloid differentiation factor 88 in resident peritoneal macrophages. However, in bone marrow-derived macrophages, this type of competition is greatly minimized due to the lack of IRF5-dependent TLR signaling [79]. Another study has suggested that IRF4 controls M2 macrophage polarization by stimulating the expression of specific M2 macrophage markers in mice [9]. From the current evidence, STAT6, SOCS1/SOCS3, and IRF4 are all involved in the polarization of M2 macrophages [58, 59].

3.3. Fibrotic Phase of ALI/ARDS. Pulmonary fibrosis, a late complication of ALI/ARDS, is marked by fibroblast proliferation and excessive deposition of extracellular matrix [80]. M2 phenotype cells are involved in regulating the fibrotic responses in the lungs $[81,82]$. Persistence of M2 macrophages at the injury sites is a hallmark of the development of fibrosis, and the steady expression of IL- 4 and IL-13 can promote collagen deposition through TGF- $\beta$ and arginase 1 pathways [83, 84]. Wakayama et al. [85] have demonstrated that dental pulp stem cells can ameliorate bleomycin- 
induced lung injury and fibrosis by inducing antiinflammatory M2-like lung macrophages. Moreover, recent studies have reported that IL-4-polarized M2 macrophages have the potential to limit fibrosis through expressing arginase 1 and resistin-like $\alpha$ (surface markers of the M2 phenotype) genes $[78,86]$. In summary, macrophages are paradoxically involved in both the generation of pulmonary fibrosis and the later healing process [86]. They regulate fibroblast recruitment, growth, and connective-tissue remodeling; in addition, they contribute to the removal of dead tissue, the growth of new blood vessels, and fibrin dissolution [61]. The regulatory mechanism for the balance of positive and negative regulators on the profibrotic functions of macrophages needs to be clarified in the future.

\section{Conclusion}

In this review, we summarize the current research on the role of macrophages/monocytes in inflammation, tissue repair, and fibrosis in ALI/ARDS. In general, macrophages/monocytes exert a proinflammatory or an anti-inflammatory effect based on the microenvironment in different stages. Limiting excessive proinflammatory responses in the exudative phase and excessive fibroblast proliferation in the repair phase through the regulation of macrophage activation and polarization may be a novel therapeutic target for ALI/ARDS. However, there is still a long way to go.

\section{Conflicts of Interest}

The authors declare that they have no competing interests.

\section{Authors' Contributions}

Xiaofang Huang and Huiqing Xiu contributed equally to this work.

\section{Acknowledgments}

This work was supported in part by grants from the National Natural Science Foundation of China (no. 81570017, Gensheng Zhang), the Medical and Health Research Program of Zhejiang Province (Core Talents Plan; no. 2016RCA014, Gensheng Zhang), and the Medical and Health Research Program of Zhejiang Province (no. 2018KY094, Shufang Zhang).

\section{References}

[1] L. B. Ware and M. A. Matthay, "The acute respiratory distress syndrome," New England Journal of Medicine, vol. 342, no. 18, pp. 1334-1349, 2000.

[2] A. P. Wheeler and G. R. Bernard, "Acute lung injury and the acute respiratory distress syndrome: a clinical review," The Lancet, vol. 369, no. 9572, pp. 1553-1564, 2007.

[3] G. M. Matuschak and A. J. Lechner, "Acute lung injury and the acute respiratory distress syndrome: pathophysiology and treatment," Missouri Medicine, vol. 107, no. 4, pp. 252-258, 2010.
[4] J. Lomas-Neira, C. S. Chung, M. Perl, S. Gregory, W. Biffl, and A. Ayala, "Role of alveolar macrophage and migrating neutrophils in hemorrhage-induced priming for ALI subsequent to septic challenge," American Journal of Physiology. Lung Cellular and Molecular Physiology, vol. 290, no. 1, pp. L51-L58, 2006.

[5] L. K. Johnston, C. R. Rims, S. E. Gill, J. K. McGuire, and A. M. Manicone, "Pulmonary macrophage subpopulations in the induction and resolution of acute lung injury," American Journal of Respiratory Cell and Molecular Biology, vol. 47, no. 4, pp. 417-426, 2012.

[6] H. Dickensheets, N. Vazquez, F. Sheikh et al., "Suppressor of cytokine signaling-1 is an IL-4-inducible gene in macrophages and feedback inhibits IL-4 signaling," Genes and Immunity, vol. 8, no. 1, pp. 21-27, 2007.

[7] Y. Liu, K. N. Stewart, E. Bishop et al., "Unique expression of suppressor of cytokine signaling 3 is essential for classical macrophage activation in rodents in vitro and in vivo," Journal of Immunology, vol. 180, no. 9, pp. 6270-6278, 2008.

[8] T. Krausgruber, K. Blazek, T. Smallie et al., "IRF5 promotes inflammatory macrophage polarization and $\mathrm{T}_{\mathrm{H}} 1-\mathrm{T}_{\mathrm{H}} 17$ responses," Nature Immunology, vol. 12, no. 3, pp. 231-238, 2011.

[9] T. Satoh, O. Takeuchi, A. Vandenbon et al., "The Jmjd3-Irf4 axis regulates $\mathrm{M} 2$ macrophage polarization and host responses against helminth infection," Nature Immunology, vol. 11, no. 10, pp. 936-944, 2010.

[10] A. Sica and A. Mantovani, "Macrophage plasticity and polarization: in vivo veritas," The Journal of Clinical Investigation, vol. 122, no. 3, pp. 787-795, 2012.

[11] K. R. Short, E. J. B. V. Kroeze, R. A. M. Fouchier, and T. Kuiken, "Pathogenesis of influenza-induced acute respiratory distress syndrome," The Lancet Infectious Diseases, vol. 14, no. 1, pp. 57-69, 2014.

[12] M. Duan, W. C. Li, R. Vlahos, M. J. Maxwell, G. P. Anderson, and M. L. Hibbs, "Distinct macrophage subpopulations characterize acute infection and chronic inflammatory lung disease," Journal of Immunology, vol. 189, no. 2, pp. 946-955, 2012.

[13] N. R. Aggarwal, L. S. King, and F. R. D'Alessio, "Diverse macrophage populations mediate acute lung inflammation and resolution," American Journal of Physiology. Lung Cellular and Molecular Physiology, vol. 306, no. 8, pp. L709-L725, 2014.

[14] S. Herold, N. M. Gabrielli, and I. Vadasz, "Novel concepts of acute lung injury and alveolar-capillary barrier dysfunction," American Journal of Physiology. Lung Cellular and Molecular Physiology, vol. 305, no. 10, pp. L665-L681, 2013.

[15] N. van Rooijen, R. van Nieuwmegen, and E. W. A. Kamperdijk, "Elimination of phagocytic cells in the spleen after intravenous injection of liposomeencapsulated dichloromethylene diphosphonate. Ultrastructural aspects of elimination of marginal zone macrophages," Virchows Archiv B Cell Pathology Including Molecular Pathology, vol. 49, no. 1, pp. 375-383, 1985.

[16] N. van Rooijen, N. Kors, M. Ende, and C. D. Dijkstra, "Depletion and repopulation of macrophages in spleen and liver of rat after intravenous treatment with liposome-encapsulated dichloromethylene diphosphonate," Cell and Tissue Research, vol. 260, no. 2, pp. 215-222, 1990.

[17] J. A. Frank, C. M. Wray, D. F. McAuley, R. Schwendener, and M. A. Matthay, "Alveolar macrophages contribute to alveolar 
barrier dysfunction in ventilator-induced lung injury," American Journal of Physiology. Lung Cellular and Molecular Physiology, vol. 291, no. 6, pp. L1191-L1198, 2006.

[18] F. G. Eyal, C. R. Hamm, and J. C. Parker, "Reduction in alveolar macrophages attenuates acute ventilator induced lung injury in rats," Intensive Care Medicine, vol. 33, no. 7, pp. 1212-1218, 2007.

[19] M. A. Koay, X. Gao, M. K. Washington et al., "Macrophages are necessary for maximal nuclear factor- $\kappa \mathrm{B}$ activation in response to endotoxin," American Journal of Respiratory Cell and Molecular Biology, vol. 26, no. 5, pp. 572-578, 2002.

[20] D. Machado-Aranda, M. V. Suresh, B. Yu, V. Dolgachev, M. R. Hemmila, and K. Raghavendran, "Alveolar macrophage depletion increases the severity of acute inflammation following nonlethal unilateral lung contusion in mice," Journal of Trauma and Acute Care Surgery, vol. 76, no. 4, pp. 982-990, 2014.

[21] E. Broug-Holub, G. B. Toews, J. van Iwaarden et al., “Alveolar macrophages are required for protective pulmonary defenses in murine Klebsiella pneumonia: elimination of alveolar macrophages increases neutrophil recruitment but decreases bacterial clearance and survival," Infection and Immunity, vol. 65, no. 4, pp. 1139-1146, 1997.

[22] T. Narasaraju, E. Yang, R. P. Samy et al., "Excessive neutrophils and neutrophil extracellular traps contribute to acute lung injury of influenza pneumonitis," The American Journal of Pathology, vol. 179, no. 1, pp. 199-210, 2011.

[23] A. E. Williams and R. C. Chambers, "The mercurial nature of neutrophils: still an enigma in ARDS?," American Journal of Physiology. Lung Cellular and Molecular Physiology, vol. 306, no. 3, pp. L217-L230, 2014.

[24] A. J. Duffy, B. Nolan, K. Sheth, H. Collette, M. De, and P. E. Bankey, "Inhibition of alveolar neutrophil immigration in endotoxemia is macrophage inflammatory protein 2 independent," The Journal of Surgical Research, vol. 90, no. 1, pp. 51$57,2000$.

[25] J. B. Zamjahn, L. J. Quinton, J. C. Mack, C. W. Frevert, S. Nelson, and G. J. Bagby, "Differential flux of macrophage inflammatory protein-2 and cytokine-induced neutrophil chemoattractant from the lung after intrapulmonary delivery," American Journal of Physiology. Lung Cellular and Molecular Physiology, vol. 301, no. 4, pp. L568-L574, 2011.

[26] S. C. Donnelly, C. Haslett, R. M. Strieter et al., "Interleukin-8 and development of adult respiratory distress syndrome in at-risk patient groups," The Lancet, vol. 341, no. 8846, pp. 643-647, 1993.

[27] E. R. Chilvers, K. A. Cadwallader, B. J. Reed, J. F. White, and A. M. Condliffe, "The function and fate of neutrophils at the inflamed site: prospects for therapeutic intervention," Journal of the Royal College of Physicians of London, vol. 34, no. 1, pp. 68-74, 2000.

[28] S. J. Webster, M. Daigneault, M. A. Bewley et al., "Distinct cell death programs in monocytes regulate innate responses following challenge with common causes of invasive bacterial disease," Journal of Immunology, vol. 185, no. 5, pp. 29682979, 2010.

[29] H. Amano, K. Morimoto, M. Senba et al., "Essential contribution of monocyte chemoattractant protein-1/C-C chemokine ligand-2 to resolution and repair processes in acute bacterial pneumonia," Journal of Immunology, vol. 172, no. 1, pp. 398-409, 2004.
[30] D. H. Seitz, U. Niesler, A. Palmer et al., "Blunt chest trauma induces mediator-dependent monocyte migration to the lung," Critical Care Medicine, vol. 38, no. 9, pp. 1852-1859, 2010.

[31] P. J. Christensen, M. du, B. Moore, S. Morris, G. B. Toews, and R. Paine III, "Expression and functional implications of CCR2 expression on murine alveolar epithelial cells," American Journal of Physiology. Lung Cellular and Molecular Physiology, vol. 286, no. 1, pp. L68-L72, 2004.

[32] S. Herold, T. S. Tabar, H. Janßen et al., "Exudate macrophages attenuate lung injury by the release of IL-1 receptor antagonist in gram-negative pneumonia," American Journal of Respiratory and Critical Care Medicine, vol. 183, no. 10, pp. 13801390, 2011.

[33] K. Dhaliwal, E. Scholefield, D. Ferenbach et al., "Monocytes control second-phase neutrophil emigration in established lipopolysaccharide-induced murine lung injury," American Journal of Respiratory and Critical Care Medicine, vol. 186, no. 6, pp. 514-524, 2012.

[34] K. J. Mould, L. Barthel, M. P. Mohning et al., "Cell origin dictates programming of resident versus recruited macrophages during acute lung injury," American Journal of Respiratory Cell and Molecular Biology, vol. 57, no. 3, pp. 294-306, 2017.

[35] S. Herold, K. Mayer, and J. Lohmeyer, "Acute lung injury: how macrophages orchestrate resolution of inflammation and tissue repair," Frontiers in Immunology, vol. 2, p. 65, 2011.

[36] S. Arora, K. Dev, B. Agarwal, P. Das, and M. A. Syed, "Macrophages: their role, activation and polarization in pulmonary diseases," Immunobiology, vol. 223, no. 4-5, pp. 383-396, 2018.

[37] P. R. Taylor, L. Martinez-Pomares, M. Stacey, H. H. Lin, G. D. Brown, and S. Gordon, "Macrophage receptors and immune recognition," Annual Review of Immunology, vol. 23, no. 1, pp. 901-944, 2005.

[38] D. M. Higgins, J. Sanchez-Campillo, A. G. Rosas-Taraco et al., "Relative levels of M-CSF and GM-CSF influence the specific generation of macrophage populations during infection with Mycobacterium tuberculosis," Journal of Immunology, vol. 180, no. 7, pp. 4892-4900, 2008.

[39] M. R. Wilson, S. Choudhury, M. E. Goddard, K. P. O'Dea, A. G. Nicholson, and M. Takata, "High tidal volume upregulates intrapulmonary cytokines in an in vivo mouse model of ventilator-induced lung injury," Journal of Applied Physiology, vol. 95, no. 4, pp. 1385-1393, 2003.

[40] B. Beck-Schimmer, R. Schwendener, T. Pasch, L. Reyes, C. Booy, and R. C. Schimmer, "Alveolar macrophages regulate neutrophil recruitment in endotoxin-induced lung injury," Respiratory Research, vol. 6, no. 1, p. 61, 2005.

[41] M. Bhatia, R. L. Zemans, and S. Jeyaseelan, "Role of chemokines in the pathogenesis of acute lung injury," American Journal of Respiratory Cell and Molecular Biology, vol. 46, no. 5, pp. 566-572, 2012.

[42] E. Vergadi, K. Vaporidi, E. E. Theodorakis et al., "Akt2 deficiency protects from acute lung injury via alternative macrophage activation and miR-146a induction in mice," Journal of Immunology, vol. 192, no. 1, pp. 394-406, 2013.

[43] J. E. Da Silva-Santos, M. C. Santos-Silva, Q. Cunha Fde, and J. Assreuy, "The role of ATP-sensitive potassium channels in neutrophil migration and plasma exudation," The Journal of Pharmacology and Experimental Therapeutics, vol. 300, no. 3, pp. 946-951, 2002. 
[44] Y. Xu, C. Meng, G. Liu et al., "Classically activated macrophages protect against lipopolysaccharide-induced acute lung injury by expressing amphiregulin in mice," Anesthesiology, vol. 124, no. 5, pp. 1086-1099, 2016.

[45] T. Dolinay, N. Kaminski, M. Felgendreher et al., "Gene expression profiling of target genes in ventilator-induced lung injury," Physiological Genomics, vol. 26, no. 1, pp. 68-75, 2006.

[46] C. M. Minutti, B. García-Fojeda, A. Sáenz et al., "Surfactant protein A prevents IFN- $\gamma / \mathrm{IFN}-\gamma$ receptor interaction and attenuates classical activation of human alveolar macrophages," Journal of Immunology, vol. 197, no. 2, pp. 590-598, 2016.

[47] M. A. Meraz, J. M. White, K. C. F. Sheehan et al., "Targeted disruption of the Stat1 gene in mice reveals unexpected physiologic specificity in the JAK-STAT signaling pathway," Cell, vol. 84, no. 3, pp. 431-442, 1996.

[48] J. E. Durbin, R. Hackenmiller, M. C. Simon, and D. E. Levy, "Targeted disruption of the mouse Stat1 gene results in compromised innate immunity to viral disease," Cell, vol. 84, no. 3, pp. 443-450, 1996.

[49] W. S. Alexander and D. J. Hilton, "The role of suppressors of cytokine signaling (SOCS) proteins in regulation of the immune response," Annual Review of Immunology, vol. 22, no. 1, pp. 503-529, 2004.

[50] H. Yasukawa, A. Sasaki, and A. Yoshimura, "Negative regulation of cytokine signaling pathways," Annual Review of Immunology, vol. 18, no. 1, pp. 143-164, 2000.

[51] E. M. Linossi, J. J. Babon, D. J. Hilton, and S. E. Nicholson, "Suppression of cytokine signaling: the SOCS perspective," Cytokine \& Growth Factor Reviews, vol. 24, no. 3, pp. 241248, 2013.

[52] R. Nakagawa, T. Naka, H. Tsutsui et al., "SOCS-1 participates in negative regulation of LPS responses," Immunity, vol. 17, no. 5, pp. 677-687, 2002.

[53] A. Baetz, M. Frey, K. Heeg, and A. H. Dalpke, "Suppressor of cytokine signaling (SOCS) proteins indirectly regulate tolllike receptor signaling in innate immune cells," The Journal of Biological Chemistry, vol. 279, no. 52, pp. 54708-54715, 2004.

[54] Y. B. Liang, H. Tang, Z. B. Chen et al., "Downregulated SOCS1 expression activates the JAK1/STAT1 pathway and promotes polarization of macrophages into M1 type," Molecular Medicine Reports, vol. 16, no. 5, pp. 6405-6411, 2017.

[55] H. Qin, A. T. Holdbrooks, Y. Liu, S. L. Reynolds, L. L. Yanagisawa, and E. N. Benveniste, "SOCS3 deficiency promotes M1 macrophage polarization and inflammation," Journal of Immunology, vol. 189, no. 7, pp. 3439-3448, 2012.

[56] K. Sun, S. B. He, J. G. Qu et al., "IRF5 regulates lung macrophages M2 polarization during severe acute pancreatitis in vitro," World Journal of Gastroenterology, vol. 22, no. 42, pp. 9368-9377, 2016.

[57] H. Qin, W. I. Yeh, P. de Sarno et al., "Signal transducer and activator of transcription-3/suppressor of cytokine signaling3 (STAT3/SOCS3) axis in myeloid cells regulates neuroinflammation," Proceedings of the National Academy of Sciences of the United States of America, vol. 109, no. 13, pp. 5004-5009, 2012.

[58] S. Gordon and F. O. Martinez, "Alternative activation of macrophages: mechanism and functions," Immunity, vol. 32, no. 5, pp. 593-604, 2010.

[59] K. Honda and T. Taniguchi, "IRFs: master regulators of signalling by toll-like receptors and cytosolic pattern-recognition receptors," Nature Reviews. Immunology, vol. 6, no. 9, pp. 644-658, 2006.

[60] A. Androulidaki, D. Iliopoulos, A. Arranz et al., "The kinase Aktl controls macrophage response to lipopolysaccharide by regulating microRNAs," Immunity, vol. 31, no. 2, pp. 220 231, 2009.

[61] S. Gordon, "Alternative activation of macrophages," Nature Reviews Immunology, vol. 3, no. 1, pp. 23-35, 2003.

[62] S. Colin, G. Chinetti-Gbaguidi, and B. Staels, "Macrophage phenotypes in atherosclerosis," Immunological Reviews, vol. 262, no. 1, pp. 153-166, 2014.

[63] K. Suzuki, K. Meguro, D. Nakagomi, and H. Nakajima, "Roles of alternatively activated M2 macrophages in allergic contact dermatitis," Allergology International, vol. 66, no. 3, pp. 392 397, 2017.

[64] P. M. Henson and D. A. Hume, "Apoptotic cell removal in development and tissue homeostasis," Trends in Immunology, vol. 27, no. 5, pp. 244-250, 2006.

[65] M. R. Elliott, K. M. Koster, and P. S. Murphy, "Efferocytosis signaling in the regulation of macrophage inflammatory responses," Journal of Immunology, vol. 198, no. 4, pp. 13871394, 2017.

[66] S. Kim, K. B. Elkon, and X. Ma, “Transcriptional suppression of interleukin-12 gene expression following phagocytosis of apoptotic cells," Immunity, vol. 21, no. 5, pp. 643-653, 2004.

[67] A. Byrne and D. J. Reen, "Lipopolysaccharide induces rapid production of IL-10 by monocytes in the presence of apoptotic neutrophils," Journal of Immunology, vol. 168, no. 4, pp. 1968 1977, 2002.

[68] A. Ariel and C. N. Serhan, "New lives given by cell death: macrophage differentiation following their encounter with apoptotic leukocytes during the resolution of inflammation," Frontiers in Immunology, vol. 3, p. 4, 2012.

[69] D. Korns, S. C. Frasch, R. Fernandez-Boyanapalli, P. M. Henson, and D. L. Bratton, "Modulation of macrophage efferocytosis in inflammation," Frontiers in Immunology, vol. 2, p. 57, 2011.

[70] O. E. Akilov, M. X. Wu, Y. Jin et al., "Vaccination with photodynamic therapy-treated macrophages induces highly suppressive T-regulatory cells," Photodermatology, Photoimmunology \& Photomedicine, vol. 27, no. 2, pp. 97-107, 2011.

[71] O. Boenisch, M. Lopez, W. Elyaman, C. N. Magee, U. Ahmad, and N. Najafian, "Ex vivo expansion of human Tregs by rabbit ATG is dependent on intact STAT3signaling in $\mathrm{CD} 4^{+} \mathrm{T}$ cells and requires the presence of monocytes," American Journal of Transplantation, vol. 12, no. 4, pp. 856-866, 2012.

[72] G. Liu, H. Ma, L. Qiu et al., "Phenotypic and functional switch of macrophages induced by regulatory $\mathrm{CD} 4{ }^{+} \mathrm{CD} 25^{+} \mathrm{T}$ cells in mice," Immunology \& Cell Biology, vol. 89, no. 1, pp. 130$142,2011$.

[73] M. M. Tiemessen, A. L. Jagger, H. G. Evans, M. J. C. van Herwijnen, S. John, and L. S. Taams, "CD $4^{+} \mathrm{CD} 25^{+} \mathrm{Foxp}^{+}$ regulatory $\mathrm{T}$ cells induce alternative activation of human monocytes/macrophages," Proceedings of the National Academy of Sciences of the United States of America, vol. 104, no. 49, pp. 19446-19451, 2007.

[74] L. S. Taams, J. M. R. van Amelsfort, M. M. Tiemessen et al., "Modulation of monocyte/macrophage function by human CD4+CD25+ regulatory T cells," Human Immunology, vol. 66 , no. 3, pp. 222-230, 2005. 
[75] F. R. D’Alessio, K. Tsushima, N. R. Aggarwal et al., "CD4 ${ }^{+-}$ $\mathrm{CD} 25^{+} \mathrm{Foxp}^{+}$Tregs resolve experimental lung injury in mice and are present in humans with acute lung injury," The Journal of Clinical Investigation, vol. 119, no. 10, pp. 2898-2913, 2009.

[76] J. J. O'Shea and W. E. Paul, "Mechanisms underlying lineage commitment and plasticity of helper $\mathrm{CD}^{+}{ }^{+} \mathrm{T}$ cells," Science, vol. 327, no. 5969, pp. 1098-1102, 2010.

[77] N. M. Heller, X. Qi, I. S. Junttila et al., “Type I IL-4Rs selectively activate IRS-2 to induce target gene expression in macrophages," Science Signaling, vol. 1, no. 51, article ra17, 2008.

[78] F. R. D'Alessio, J. M. Craig, B. D. Singer et al., "Enhanced resolution of experimental ARDS through IL-4-mediated lung macrophage reprogramming," American Journal of Physiology. Lung Cellular and Molecular Physiology, vol. 310, no. 8, pp. L733-L746, 2016.

[79] H. Negishi, Y. Ohba, H. Yanai et al., "Negative regulation of toll-like-receptor signaling by IRF-4," Proceedings of the National Academy of Sciences of the United States of America, vol. 102, no. 44, pp. 15989-15994, 2005.

[80] B. T. Thompson, R. C. Chambers, and K. D. Liu, "Acute respiratory distress syndrome," The New England Journal of Medicine, vol. 377, no. 19, pp. 1904-1905, 2017.

[81] D. V. Pechkovsky, A. Prasse, F. Kollert et al., "Alternatively activated alveolar macrophages in pulmonary fibrosismediator production and intracellular signal transduction," Clinical Immunology, vol. 137, no. 1, pp. 89-101, 2010.

[82] M. A. Gibbons, A. C. MacKinnon, P. Ramachandran et al., "Ly6C $\mathrm{C}$ monocytes direct alternatively activated profibrotic macrophage regulation of lung fibrosis," American Journal of Respiratory and Critical Care Medicine, vol. 184, no. 5, pp. 569-581, 2011.

[83] N. Duru, B. Wolfson, and Q. Zhou, "Mechanisms of the alternative activation of macrophages and non-coding RNAs in the development of radiation-induced lung fibrosis," World Journal of Biological Chemistry, vol. 7, no. 4, pp. 231239, 2016

[84] A. L. Mora, E. Torres-González, M. Rojas et al., “Activation of alveolar macrophages via the alternative pathway in herpesvirus-induced lung fibrosis," American Journal of Respiratory Cell and Molecular Biology, vol. 35, no. 4, pp. 466-473, 2006.

[85] H. Wakayama, N. Hashimoto, Y. Matsushita et al., "Factors secreted from dental pulp stem cells show multifaceted benefits for treating acute lung injury in mice," Cytotherapy, vol. 17, no. 8, pp. 1119-1129, 2015.

[86] J. T. Pesce, T. R. Ramalingam, M. M. Mentink-Kane et al., "Arginase-1-expressing macrophages suppress Th2 cytokinedriven inflammation and fibrosis," PLoS Pathogens, vol. 5, no. 4, article e1000371, 2009. 




The Scientific World Journal
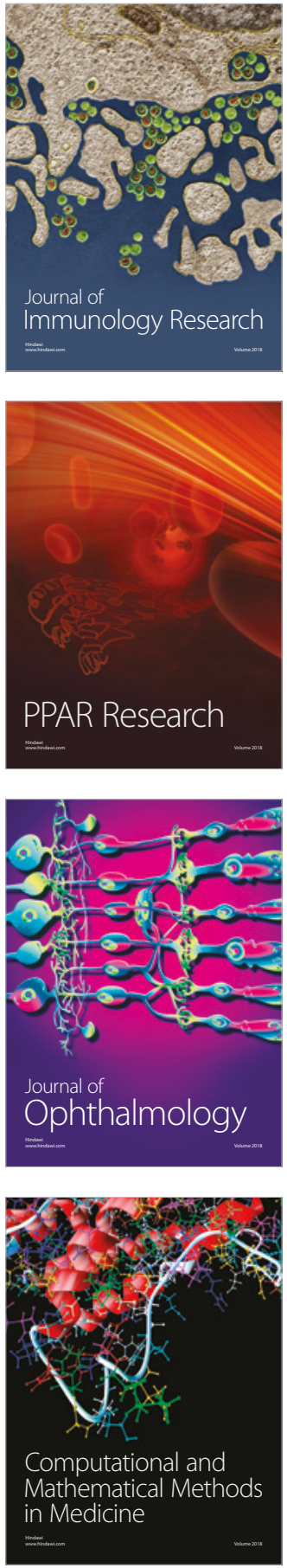

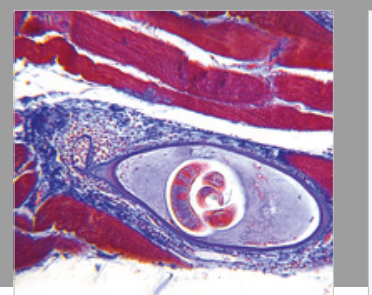

Gastroenterology Research and Practice

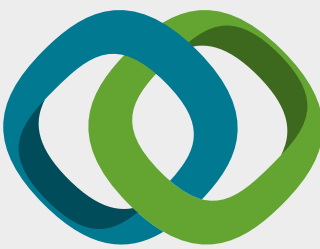

\section{Hindawi}

Submit your manuscripts at

www.hindawi.com
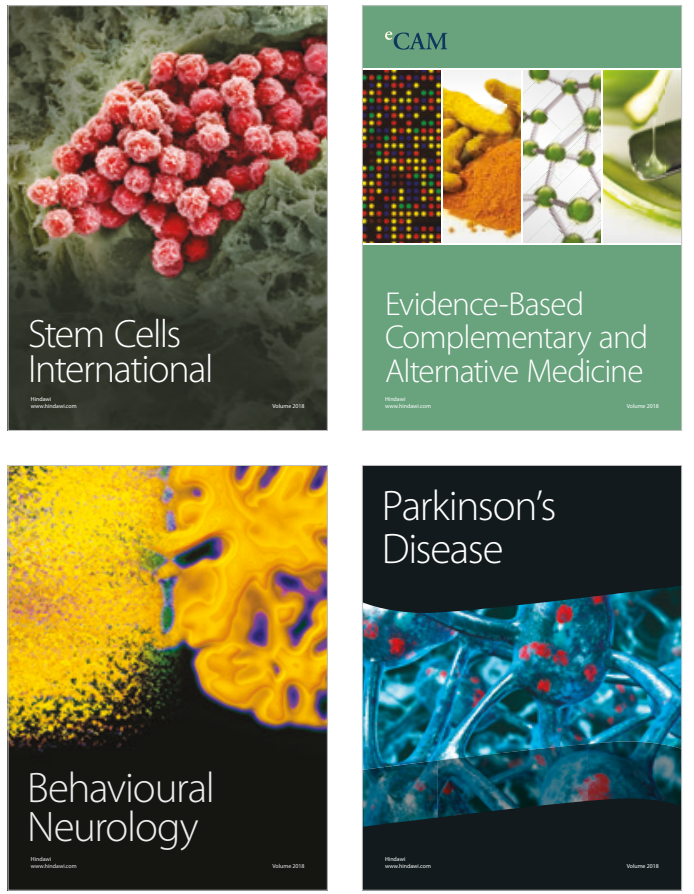



ournal of

Diabetes Research



Disease Markers
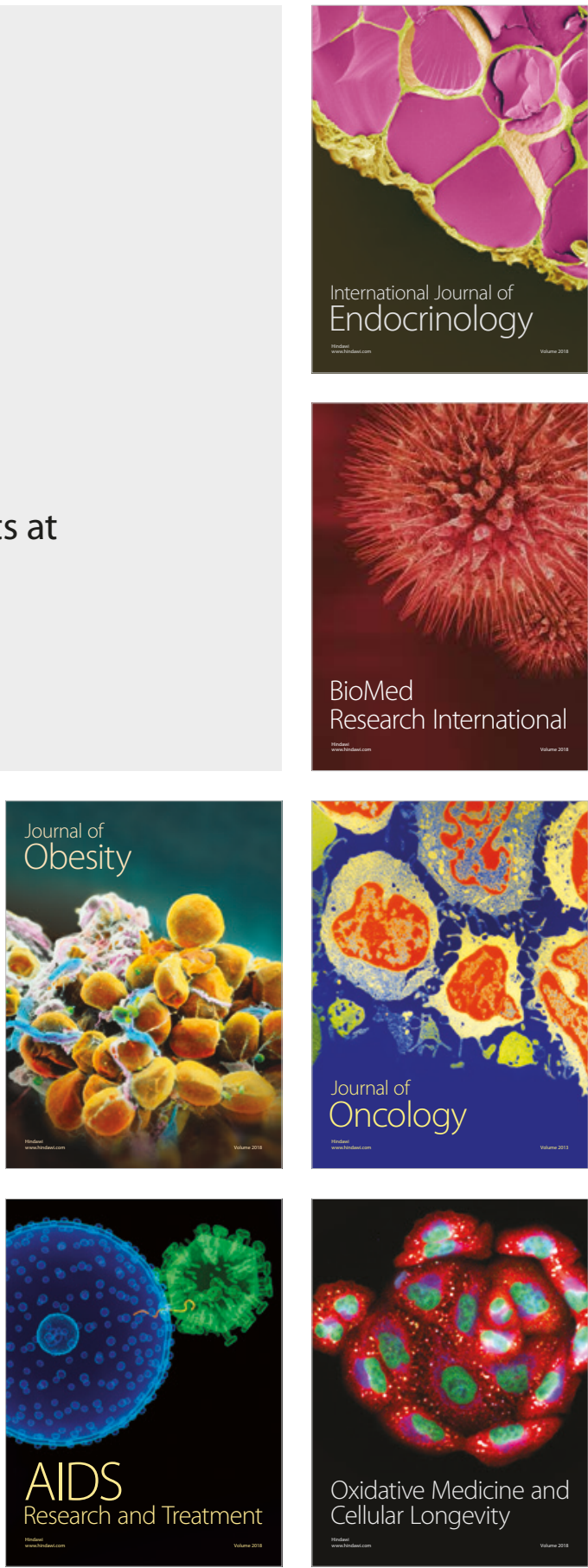